\title{
Cloud-assisted QoE guarantee scheme based on adaptive cross-layer perceptron of artificial neural network for mobile Internet
}

\author{
Zhou Silin
}

\begin{abstract}
For improving the system performance of mobile Internet, how to provide the Quality of Experience (QoE) guarantee is an important factor. First, based on artificial neural network and adaptive cross-layer perceptron, we studied the cloudassisted QoE guarantee mechanism. Then, according to the power, we divided the distance and perceptron layers of mobile Internet and cloud into three levels. We showed the state information definition of the mobile node on the basis of the adaptive adjustment perceptron layers. Thirdly, the perceptron network topology would be updated according to the customer service, which would be updated based on the perceptron learning rule for improving the training practice efficiency. The above scheme would guarantee the QoE effectively. The experimental results show that the proposed QoE guarantee mechanism has obvious advantages in terms of throughput, efficiency, and reliability.
\end{abstract}

\section{Introduction}

Mobile crowding networks can fully study the underlying data service node of the mobile communication system, which could provide better quality assurance for data communications and make full use of the sensing region mobile node communication resources, and is used in various fields, such as metro networks [1], organelle networks [2], and aqueous hydroxyapatite-gelatin networks [3]. However, how to motivate users to actively join the mobile-aware network [4] and update the network mobile node [5] becomes the key issue.

One the hand, Oh Sang-Hoon [6] proposed an algorithmic-level approach, which used the multilayer perceptrons with higher order error functions. The class imbalance problem in the context of multilayer perceptron (MLP) neural networks was investigated by Castro Cristiano et al. [7]. Chaudhuri et al. [8] developed a multilayer perceptron model and compared the forecast quality with other neural networks. Mwale et al. [9] applied a combination of self-organizing maps (SOM) and multilayer perceptron artificial neural networks to the Lower Shire

Correspondence: silinzhou@163.com

School of Computer Science \& Engineering, Changshu Institute of Technology, Changshu 215500, China floodplain of Malawi for flow- and water-level forecasting, which was used to extract features from the raw data. The use of multilayer perceptron neural networks to invert dispersion curves obtained via multichannel analysis of surface waves (MASW) for shear S-wave velocity profile was proposed by Caylak and Kaftan [10]. Ouadfeul et al. [11] implanted a tentative prediction of daily geomagnetic field and storms by analyzing the International Real-Time Magnetic Observatory Network data using the artificial neural network.

On the other hand, the improvement achieved in estimating the volume of clay in the Shurijeh Reservoir Formation is described in article [12], which dealt with an application to a gas-producing well and another nonproducing well in a joint field between Iran and Turkmenistan. Taravat et al. [13] introduced and evaluated a multilayer perceptron neural network cloud mask for Meteosat Second Generation SEVIRI images. The radial basis function network and multilayer perceptron networks were investigated in article [14] for modeling urban change. Fan et al. [15] set up a multilayer perceptron neural network prediction model based on phase reconstruction, which is for carbon price to characterize its strong nonlinearity.
焦 Springer

(c) 2016 Silin. Open Access This article is distributed under the terms of the Creative Commons Attribution 4.0 International License (http://creativecommons.org/licenses/by/4.0/), which permits unrestricted use, distribution, and reproduction in any medium, provided you give appropriate credit to the original author(s) and the source, provide a link to the Creative Commons license, and indicate if changes were made. 
Thirdly, Patel Krishna et al. [16] investigate reward-loss neural response differences among 42 current cocaine users, 35 former cocaine users, and 47 healthy subjects with a functional magnetic resonance imaging monetary incentive delay task. A secure user-centric and social-aware reputation-based incentive scheme for DTNs was proposed in article [17]. The symbiotic architecture called cognitive relaying with frequency incentive for multiple primary users (CRFI-M) was studied by Nadkar et al. [18]. The design challenges of incentive mechanisms for encouraging user engagement in user-provided networks were analyzed by Iosifidis et al. [19]. The Quality of Experience (QoE) guarantee was designed for cybersecurity [20].

However, the above research results ignored the relationship between the crowding network topology and perceptron architecture of mobile nodes. Additionally, the QoE guarantee scheme was not researched in depth. Based on the results of the above researches, the cloudassisted QoE guarantee mechanism based on adaptive cross-layer perceptron of artificial neural network was proposed for mobile crowding networks.

The rest of the paper is organized as follows. Section 2 describes the adaptive cross-layer perceptron with artificial neural network. In Section 3, we design the cloudassisted QoE guarantee mechanism based on adaptive cross-layer perceptron. Simulation results are given in Section 4. Finally, we conclude the paper in Section 5.

\section{Adaptive cross-layer perceptron with artificial neural network}

According to the deployment of $n$ mobile nodes in the mobile crowding network, the mobile node state is defined as $M\left(P, D, L_{\mathrm{N}}\right)$, where $P$ denotes the transmit power, $D$ represents the distance, and $L_{\mathrm{N}}$ represents the perception layer of the mobile node.

$T$ denotes the collection delay of mobile node transmitting signals. The $n$ mobile nodes are divided into $k$ groups based on a data signal transmission delay. Based on the transmission power of the mobile crowding node, the primary divide situation of mobile nodes is shown in Fig. 1a. The formula (1) shows the relationship.

$$
\left\{\begin{array}{l}
T_{\mathrm{sys}} \leq \sum_{i=1}^{k} \sum_{j=1}^{k_{\mathrm{N}}} T^{N_{\mathrm{RT}}}{ }_{j} \\
P_{\mathrm{sys}} \geq \sum_{i=1}^{k} \sum_{j=1}^{k_{\mathrm{N}}} P^{N_{\mathrm{RT}}}{ }_{j} \\
\mathrm{TH}_{\mathrm{sys}} \geq \sum_{i=1}^{k}\left(1-P_{\mathrm{e}}\right) \mathrm{DP}_{\text {size }} N_{\mathrm{RT}}
\end{array}\right.
$$

Here, let $N_{\mathrm{RT}}$ denote the retransmission time. $P_{\mathrm{e}}$ denotes the packet error rate. $T_{\text {sys }}$ denotes the system delay. $P_{\text {sys }}$ denotes the total power of system. $\mathrm{TH}_{\text {sys }}$ denotes the throughput of system. $\mathrm{DP}_{\text {size }}$ denotes the packet size.

In space, according to the moving speed of the mobile node and the distance between the base station and the mobile node, the mobile crowding network are divided secondly, as shown in Fig. 1b, and the relationship is shown in formula (2).

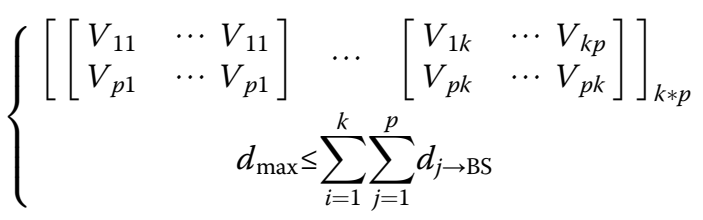

Here, let $V$ denote the moving speed. The $\mathrm{k} * \mathrm{p}$ matrix denotes the relationship between the $p$ groups of mobile nodes after second division based on the $k$ group with power. $d_{\max }$ denotes the maximum distance between the two mobile nodes. $d_{j \rightarrow \text { BS }}$ denotes the distance between the mobile node and base station.

About the perceptron layer, to optimize the mobile node perceptron learning efficiency and effectiveness of the training, the layer number of perceptron is divided based on the transmit power and spatial layout calculated within the same frequency band transmission with different distances from the mobile node input vector and perception, which satisfies the relationship of equation (3). The division is shown in Fig. 1c.

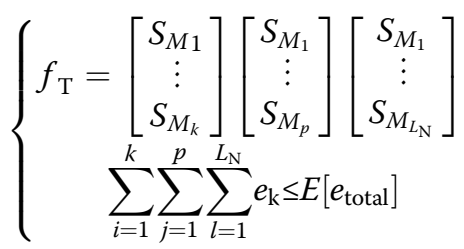

Here, $f_{\mathrm{T}}$ denotes the signal fusion which is obtained from the transmission power, the distance, and the number of the sensor layer. The fusion is used to evaluate the training effect. Let $S_{\mathrm{M} 1}$ denote the signal of mobile node. $e_{\mathrm{k}}$ denotes the learning error. $E\left[e_{\text {total }}\right]$ denotes the total learning error of mobile crowding networks.

According to the sending power, distance and layer of perceptron, and combining the network partition and moving speed, the receiving signal is given by equation (4).

$$
x_{\mathrm{S}}=\sum_{i=1}^{k} \sum_{j=1}^{p} \sum_{l=1}^{L_{\mathrm{N}}} P_{i} S_{j}\left|h_{\mathrm{d}}\right|^{2} \frac{f_{\mathrm{T}}}{E\left[e_{\mathrm{total}}\right]}
$$

Here, $h_{\mathrm{d}}$ denotes the channel fading factor between mobile node and user.

The receiving signal of neighbor mobile node is given by equation (5). 
$\Delta$

Spectrum

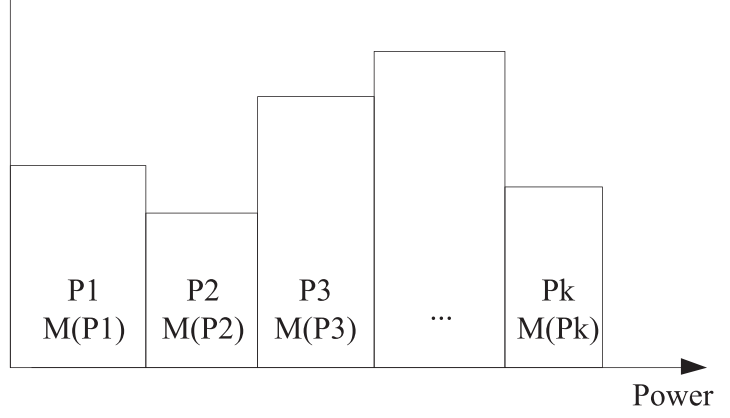

$\mathrm{P} 1+\mathrm{P} 2+\mathrm{P} 3+\cdots+\mathrm{Pk}<=$ Psys

$\sum_{i=1}^{k} M\left(P_{i}\right)=n$

a) Sending power

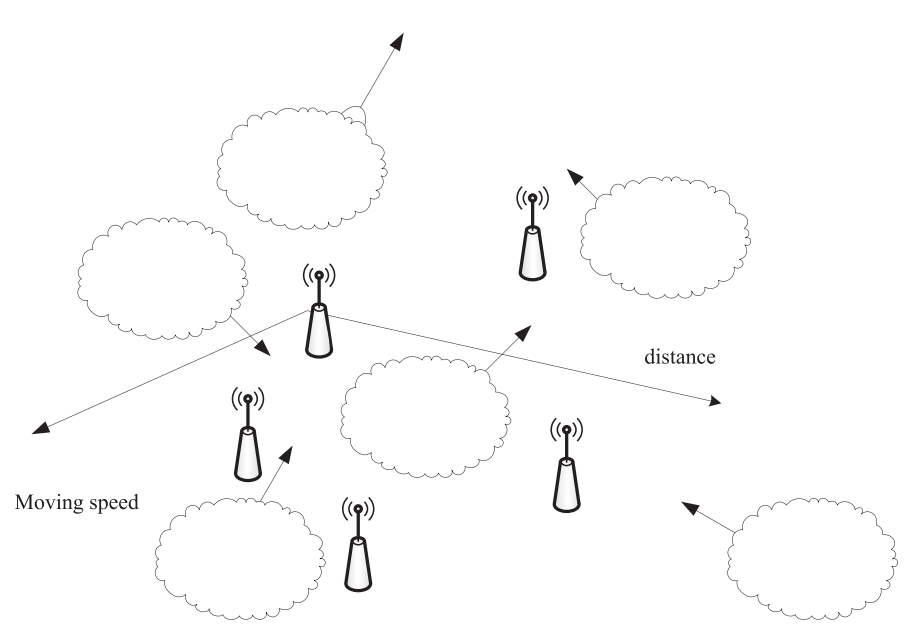

b) Distance

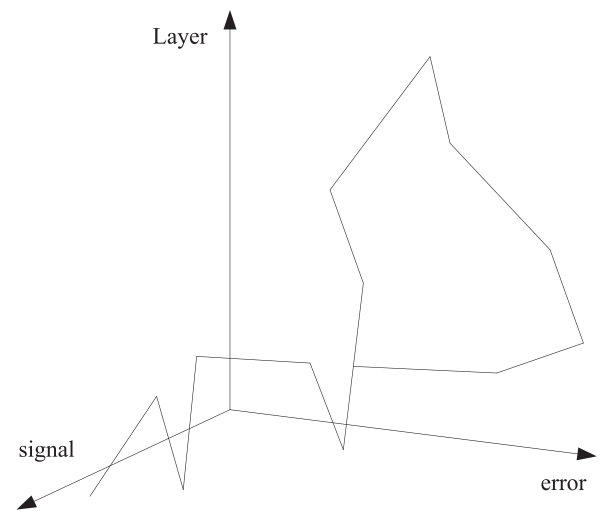

c) Layers of Perceptron

Fig. 1 Three level partition architecture. a Sending power. b distance. c layers of perceptron 


$$
y_{\mathrm{n}}=\sum_{i=1}^{k} \sum_{j=1}^{p} \sum_{l=1}^{L_{\mathrm{N}}} P_{i} S_{j}\left|h_{s \rightarrow \mathrm{user}}\right|^{2} \sum_{a=1}^{n_{\mathrm{e}}} \sqrt{P_{\mathrm{a}}} x_{\mathrm{a}}
$$

Here, $h_{s \rightarrow \text { user }}$ denotes the channel fading factor of neighbor node and receiver. $P_{\mathrm{a}}$ denotes the sending power of neighbor nodes. $x_{\mathrm{a}}$ denotes the sending signal. $n_{\mathrm{e}}$ denotes the number of the neighbor node receiving the feedback signal from the perceptron.

For optimizing the perceptron performance of mobile crowding networks and encouraging users to join the cooperation, based on artificial intelligence neural network, the perceptron would be optimized according to equations (6) and (7). This is to ensure that the transmission power, distance, and layer number of the mobile neighbor nodes could be optimized.

$$
\begin{aligned}
& P=\overbrace{\left[p_{1}, p_{2}, \ldots, p_{p}\right]}^{p} \\
& W=\overbrace{\left[w_{1}, w_{2}, \ldots, w_{L_{\mathrm{N}}}\right]}^{L_{\mathrm{N}}} \\
& \overline{\mathrm{np}}=\mathrm{np} \sum_{i=1}^{k} P_{i} p_{i} w_{i}
\end{aligned}
$$

Here, $P$ and $W$ are the input vector and target vector, respectively. np denotes the perceptron before optimization. $\overline{\mathrm{np}}$ denotes the perceptron after optimization.

The adaptive update of the cross-layer perceptron state of the mobile node can be carried out in accordance with equation (8), as shown in Fig. 2.

$$
\left\{\begin{array}{l}
\bar{P}_{\text {sys }}^{{ }_{\text {sy }}}=\alpha P_{\text {sys }} \sum_{i=1}^{k} \sum_{j=1}^{L_{\mathrm{N}}} P^{N_{\mathrm{RT}}}{ }_{j} \\
\bar{d}^{\bigotimes}=E[d] \beta \sum_{i=1}^{k} \sum_{j=1}^{L_{\mathrm{N}}} d_{j \rightarrow \mathrm{BS}} \\
E\left[\bar{e}^{\bigotimes} \text { total }\right]=\lambda \sum_{j=1}^{p} \sum_{l=1}^{L_{\mathrm{N}}} e_{k} E\left[e_{\text {total }}\right]
\end{array}\right.
$$

Here, $\alpha, \beta$, and $\gamma$ are the adaptive updating incentive weighting factors of sending power, distance, and layer number of the perceptron, respectively. To trade off between the mobile swarm intelligence network global communication performance and the user can control resources as the goal, based on three-tiered architecture, is that the cross-layer perceptron state would be adaptively updated. The analysis results of the performance are shown in Figs 3 and 4.

From Fig. 3, we found that the fitness is smaller than one with the without-adaptive update. As shown in Fig. 4, after the adaptive adjustment and updating of the sensor, the effect is better and the dividing line is clear. These results show that the proposed algorithm can be used to mobile crowding network, which has the advantages of high reliability, fast convergence, and global optimization.

\section{Cloud-assisted QoE guarantee mechanism of mobile internet}

Based on the current demand for data services, the mobile nodes of mobile crowding network can be adaptively adjusted, which would guarantee the Quality of Experience of mobile nodes. Therefore, it is particularly important to

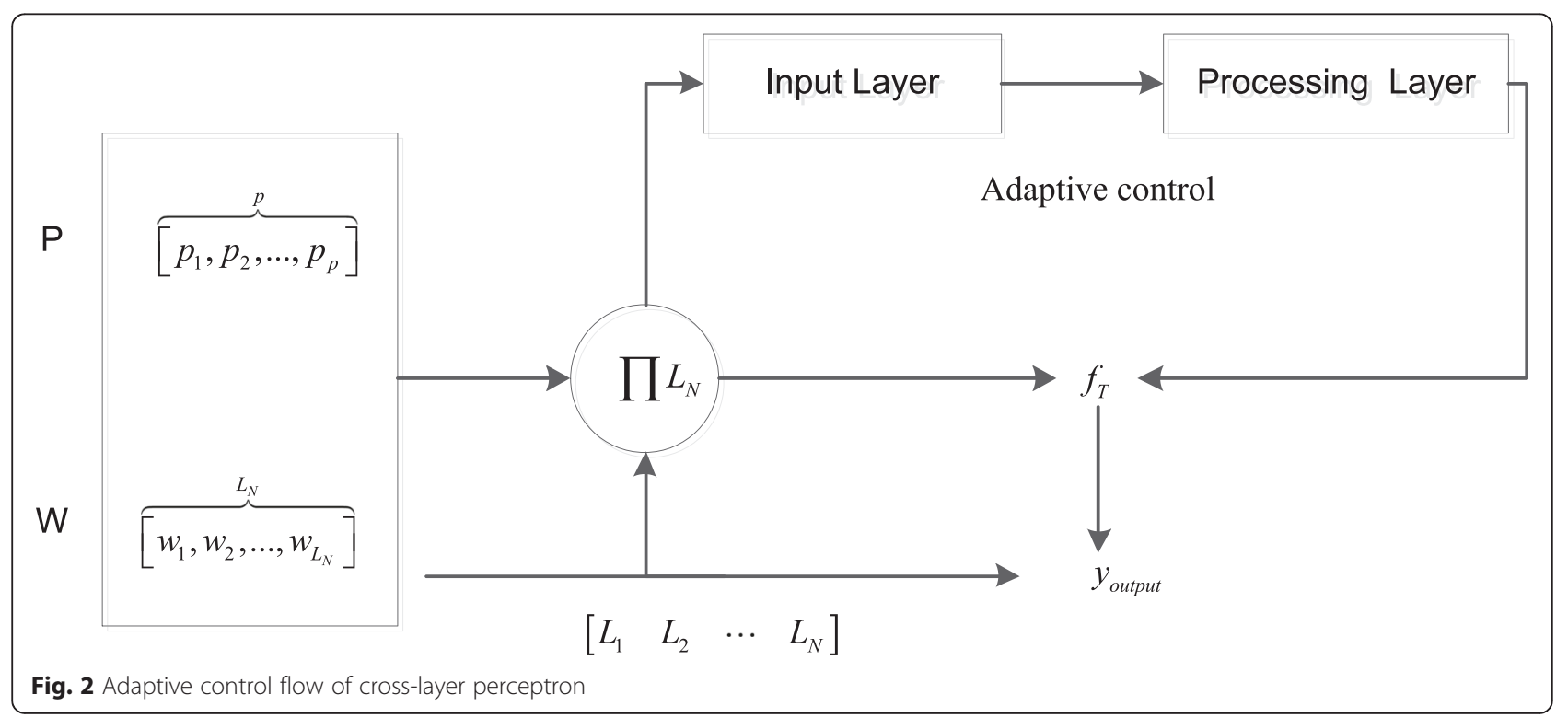




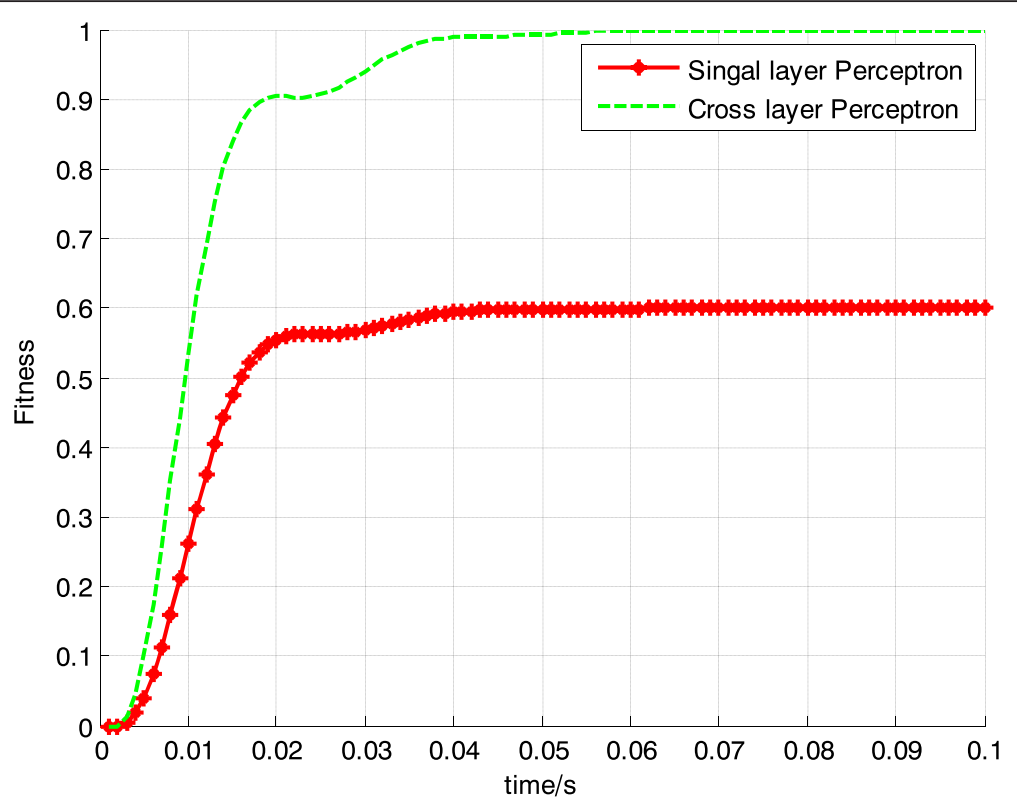

Fig. 3 Fitness

study cloud-assisted QoE guarantee mechanism with service awareness and dynamic update.

Based on the adaptive cross-layer perceptron, the cloud-assisted QoE guarantee mechanism is shown in Fig. 5. The service is launched by a base station and its users with the wireless radio, which would be released with the incentive information. The neighbor node receives the excitation signal with its state parameters. Then the perceptron would be adaptively adjust and update for constructing the service guarantee network. The initiated

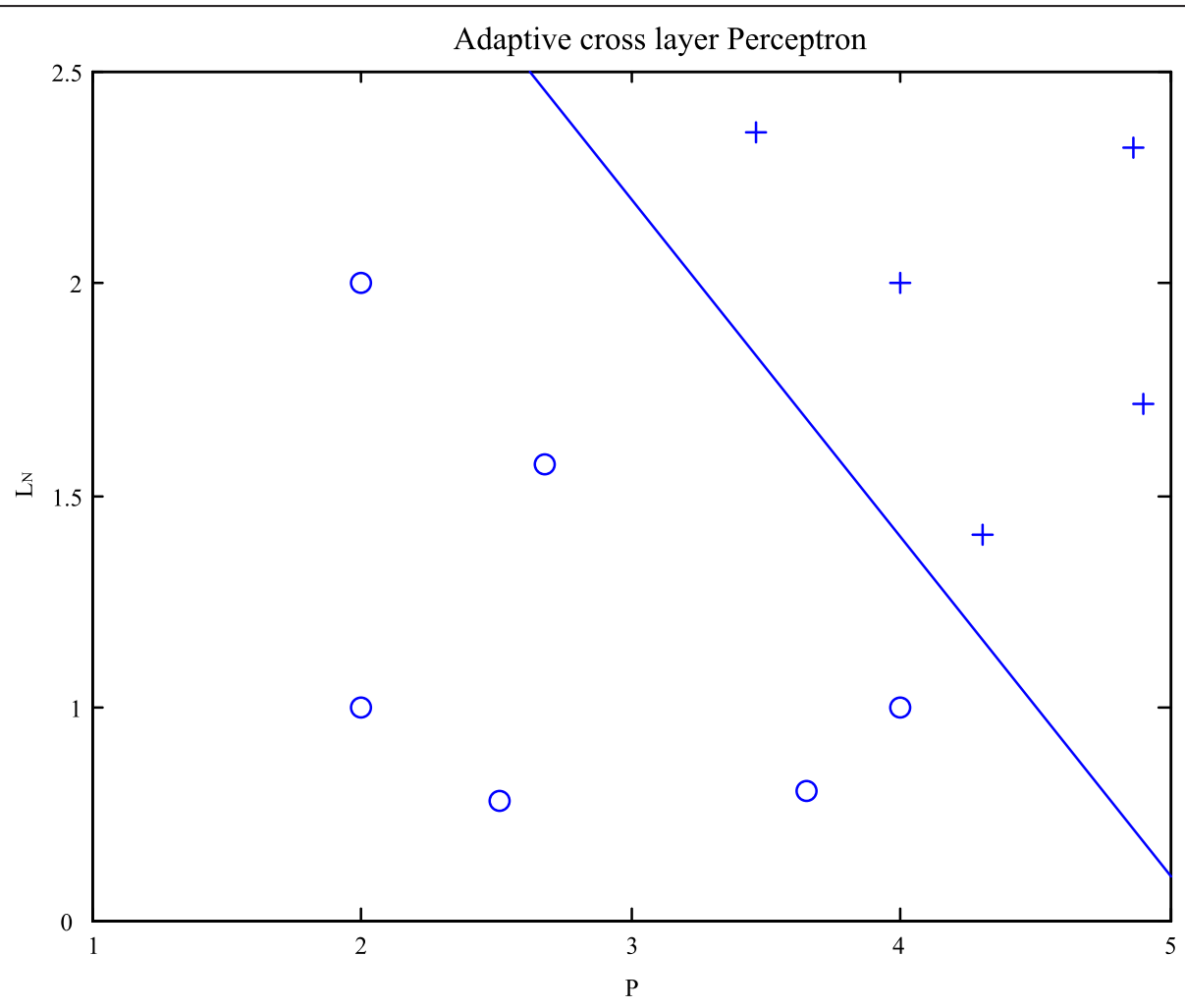

Fig. 4 Partition of crowding networks 


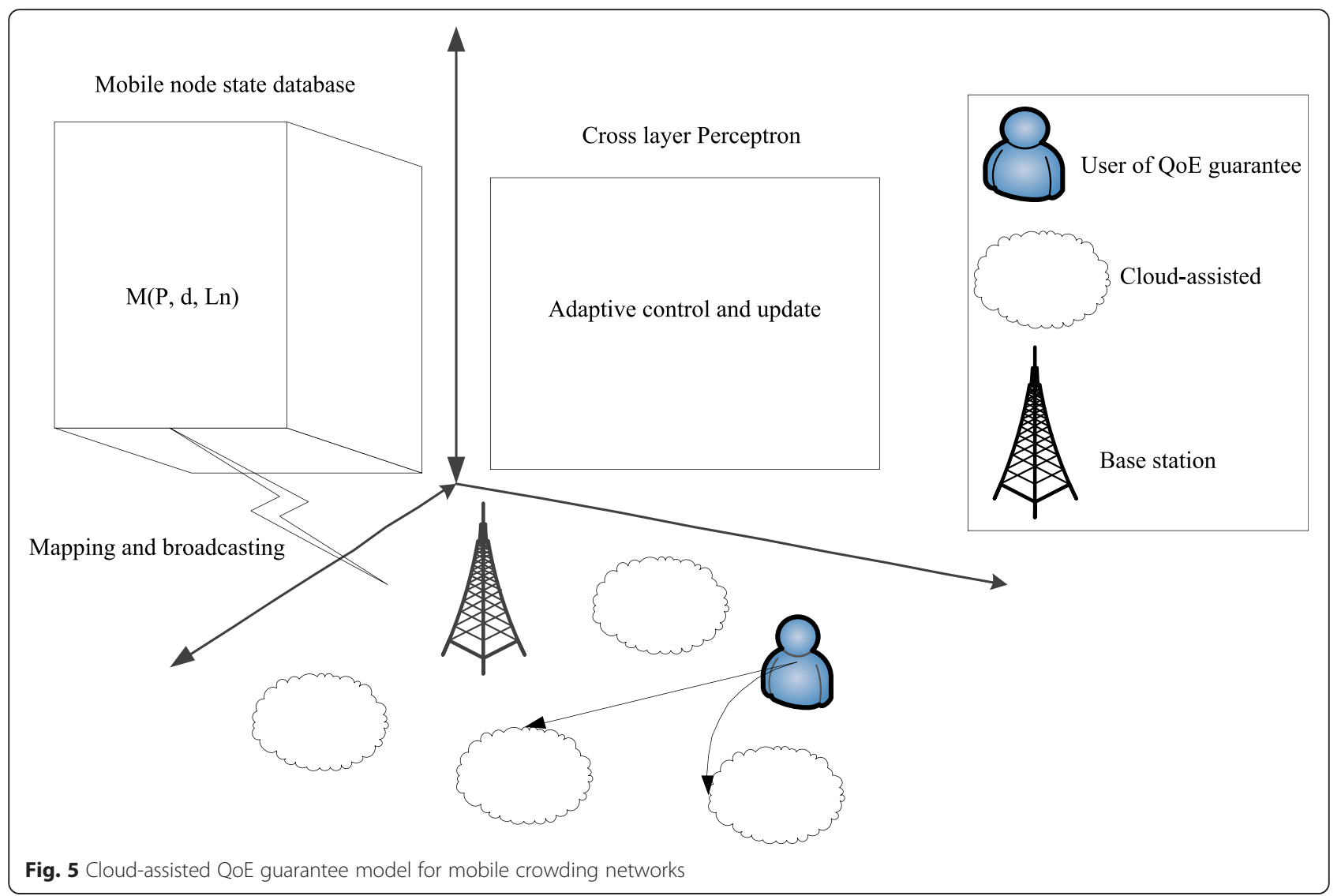

and assembly processes are shown in Fig. 5. The data transmission path is set up by the initialization, learning training, and cross-layer interaction with the perceptron of the motivated mobile nodes.

In order to effectively motivate the neighbor mobile nodes to actively join the data communication, the perceptron weights, thresholds, and perceptron layers should be real-time updated, which could be completed according to equation (9).

$$
\left\{\begin{array}{l}
\Delta p=\alpha \sum_{i=1}^{k} p_{i}+p^{N_{\mathrm{RT}}} \\
\Delta w=w\left(k, p, L_{\mathrm{N}}\right) \beta \\
\Delta L_{\mathrm{N}}=\lambda^{e_{\text {total }}} \sum_{k=1}^{p} L_{\mathrm{N}} e_{k}
\end{array}\right.
$$

Here, based on the data loss and weight factor, the power, location information, and the layer numbers should be updated in the next round, by broadcasting the full effective incentive information in mobile crowding networks. At the same time, the data transmission error should be mapped to the distance and layer number of the perceptron, which is used to avoid the waste of resources caused by data loss of the mobile node. This scheme could provide the high resource utilization rate and avoid the abandonment of the data service provider.

The mobile node state information without the cooperation communication would be adjusted and updated according to equation (10), and the perceptron would be modified and trained, waiting for the next round of tasks initiated as alternative cooperative nodes.

$$
\left\{\begin{array}{l}
\Delta w=w\left(k, p, L_{\mathrm{N}}\right)+\Delta w_{\text {Task }} \beta \\
\Delta L_{\mathrm{N}}=\Delta L_{\mathrm{N}-\text { Task }}+\lambda E\left[e_{\text {Task }}\right] \\
\text { net }=\sum_{i=1}^{q} \operatorname{PE}_{i}\left(p, w, L_{\mathrm{N}}\right)
\end{array}\right.
$$

Here, $\operatorname{PE}\left(p, w, L_{\mathrm{N}}\right)$ denotes the mobile nodes of mobile crowding networks. Let net denote the perceptron networks. The state of the mobile node is updated from the space and the layer number of the perceptron. The perceptron is updated by the $q$ iteration. The mobile state of users are random. The mobile data service nodes could be different in each round. For guaranteeing the system performance of the mobile crowding network, based on updating the perceptron network, the mobile nodes should be an effective incentive which should satisfy the conditions and performance requirements.

In order to avoid the following limitations of static perceptron: 
1. The perceptron transfer function can only accept unilateral incentives, not global optimization.

2. With nonlinear problems and classification, the efficiency is low.

3. About the face of long-time data transmission service, the number of learning iterations is higher, and the training effect is poor.

4. When the input vector and the target vector are not clear, the performance is not stable and it is easy to fluctuate.

In the process of data transmission, the active state of the mobile node creates a perceptron based on the network state and service request, as well as the initialization transfer function and learning function. A perceptron network could be trained and created according to formula (11).

$$
\left\{\begin{array}{l}
f_{\mathrm{IN}}=P_{\text {sys }} \oplus \mathrm{PW}^{k} \\
f_{\mathrm{T}}=f_{\mathrm{IN}} \oplus \mathrm{PW}^{p} \\
f_{\text {OUT }}=\sum_{i=1}^{L_{\mathrm{N}}} f^{i}{ }_{T}
\end{array}\right.
$$

Here, $f_{\mathrm{IN}}$ denotes the input vector, which could be obtained by the XOR operation of the system power and total power of user mobile node after $k$ division. $f_{\text {OUT }}$ denotes the target vector of arriving at the station.

Therefore, based on the adaptive cross-layer perceptron of artificial neural network in mobile crowding network, the cloud-assisted QoE guarantee mechanism can be divided into the following four stages: initialization of network state, division into three levels, creation of perceptron network, and cloud-assisted QoE guarantee. Among them, the creation of the perceptron network is divided into perceptron initialization, perceptron learning, and perceptron training. After the completion of the cloud-assisted QoE guarantee, the mobile crowding network is divided into active user nodes and alternative user nodes, a round of data services to provide the process of the above stages of the work flow as shown in Fig. 6.

In summary, based on a neural network and adaptive crosslayer perceptron, the mobile crowding cloud-assisted QoE guarantee mechanism (CAQG-ACL) is described as follows:

\section{Algorithm: CAQG-ACL}

Input: $M(P, d, L n),\{\alpha, \beta, \gamma\},\{p, w\}, n, m, k$

1 computing the value of $\mathrm{T}_{\text {sys }}, \mathrm{P}_{\text {sys }}, \mathrm{TH}_{\text {sys }}$

2 completing $\mathrm{k}$ group division with power

3 completing $\mathrm{p}$ group division based on primary division

4 completing thr third division based on input vector, layer number of perceptron

5 computing the value of $x_{S}, y_{n}$

6 while $\mathrm{i}<=\mathrm{n}$

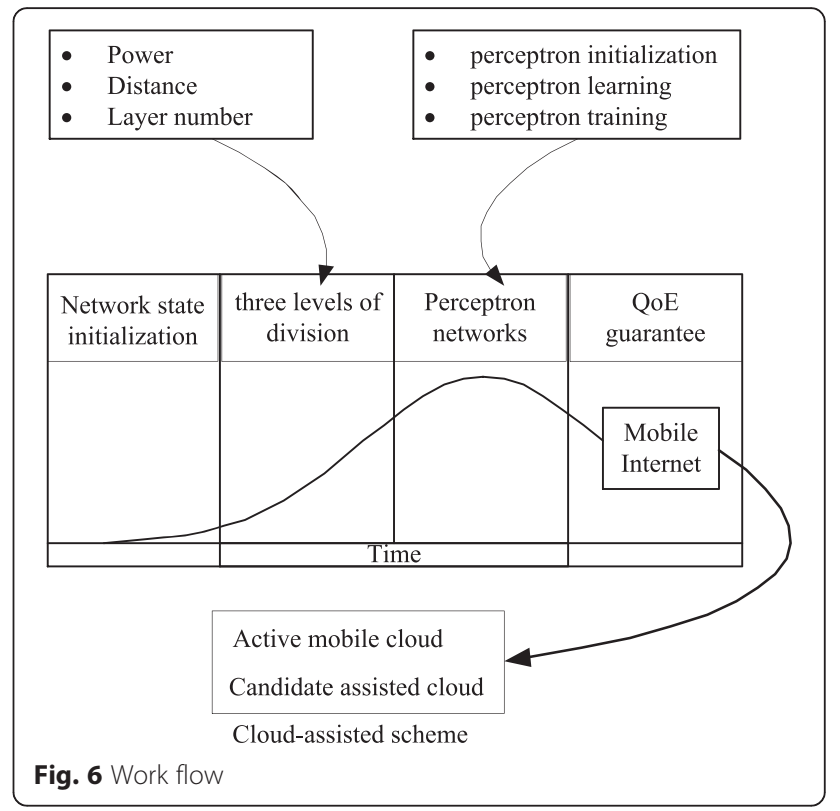

7 completing the perceptron initialization of a mobile node

$8 \mathrm{i}++$

9 end

10 while $\mathrm{i}<=\mathrm{k}$, and $\mathrm{j}<=\mathrm{p}$ and $\mathrm{l}<=\mathrm{L}_{\mathrm{N}}$

11 completing the initialization of net $=n e w p[i, j, l]$

$12 \mathrm{i}++, \mathrm{j}++, \mathrm{l++}$;

13 end

14 Developing a perceptron learning rule as PW

15 while $\mathrm{i}<=\mathrm{k}$

$16 \Delta w=w\left(k, p, L_{N}\right)+\Delta w_{\text {Task }} \beta$

$17 \Delta L_{N}=\Delta L_{N-\text { Task }}+\lambda E\left[e_{\text {Task }}\right]$

18 updating the perceptron network:

$\begin{gathered}\text { net } \\ 19 \mathrm{i}++\end{gathered}=\sum_{i=1}^{q} P E_{i}\left(p, w, L_{N}\right)$

20 end

21 end

\section{Performance evaluation}

Within a $20 \mathrm{~km} * 10 \mathrm{~km}$ wide rectangular area of mobile Internet, 3 base stations are deployed and 50 mobile nodes move randomly from 8 different angles to the region. The moving speed is from 1 to $5 \mathrm{~km} / \mathrm{h}$. There are five clouds in the assisted platform. The experimental time is $50 \mathrm{~min}$, the step size of the user-moving nodes in the region is 5 , and the number of rectangular area users reached the maximum at $50 \mathrm{~min}$ every $10 \mathrm{~min}$. In order to analyze and verify the proposed user-incentive mechanism in a mobile crowding network, we compared the throughput rate, execution efficiency, and the symbol error rate of the proposed scheme with the single-layer perceptron of QoE guarantee mechanism denoted as QG-SP. 
Figure 7 shows the results of the performance comparison between CAQG-ACL and QG-SP in three ways. Figure $7 \mathrm{a}$ gives the change of throughput rate in $50 \mathrm{~min}$. It is found that the throughput rate of the CAQG-ACL maintains a high and obvious rising trend, which benefits from a mobile node perceptron update process. The proposed scheme not only reduced user channel contention probability but also improved the success arrival rate of packet. The throughput of QG-SP increased obviously in $20 \mathrm{~min}$. But there is a big jitter after $20 \mathrm{~min}$, and the maximum throughput rate is still less than CAQG-ACL.

Figure $7 \mathrm{~b}$ shows the result of execution efficiency with mobile node scale. The execution efficiency of the proposed CAQG-ACL is about two times of the QG-SP. When the mobile node number is more than 20, execution efficiency reached $95 \%$, which further increased to $100 \%$. However, the execution efficiency of QG-SP is always hovering at $70 \%$, and the jitter is serious. Execution efficiency is guaranteed by the user's incentive mechanism in which the cross-layer interaction is updated in real time and the adaptive adjustment mechanism of the multilayer perceptron.

Figure $7 \mathrm{c}$ gives the reliability performance of the two mechanisms with time. We found that the symbol error of QG-SP decreased first and then rapidly increased before 15 min, which maintained a high symbol error rate. This is because the static sensor structure of QGSP cannot perceive the mobile node real-time status and the topology of the dynamic network, which resulted in a large number of users exiting the cooperative data transmission. However, the proposed CAQG-ACL considered the real-time status of the base station, a mobile node, and the task of initiating nodes through a three-grade classification, which has the efficient implementation of real-time optimization and updating perceptron network. Hence, the proposed scheme is more effective to motivate the user node and provide reliable data services.

\section{Conclusions}

The artificial neural network is applied to the mobile swarm intelligence network, and a highly effective reliable cloud-assisted QoE guarantee mechanism is studied. First, the crowding networks are divided with three levels, which are the power of the mobile nodes, the space location, and the number of the sensor layer. Secondly, the creation process of the sensor network was proposed, including the sensor initialization, the perceptron learning, and the perceptron training. After the completion of the cloud-assisted QoE guarantee, the mobile crowding network is divided into active user nodes and alternative user nodes. According to the user's needs, the real-time state of the perceptron network, and crowding network, the cloudassisted QoE guarantee mechanism is put forward.

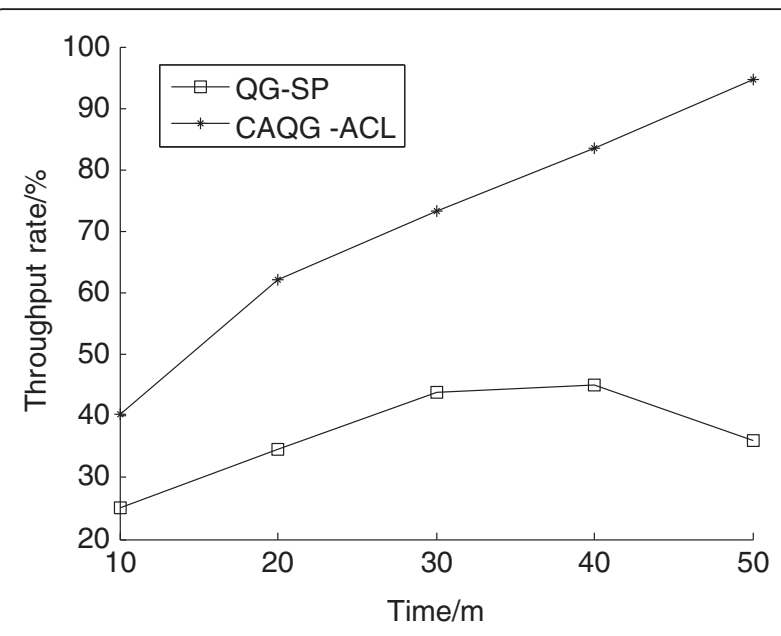

a) Throughput rate

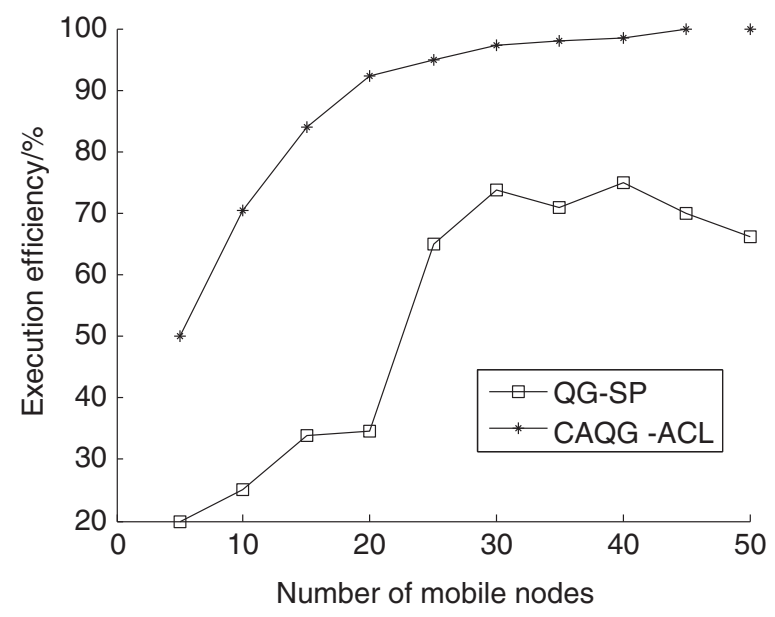

b) Execution efficiency

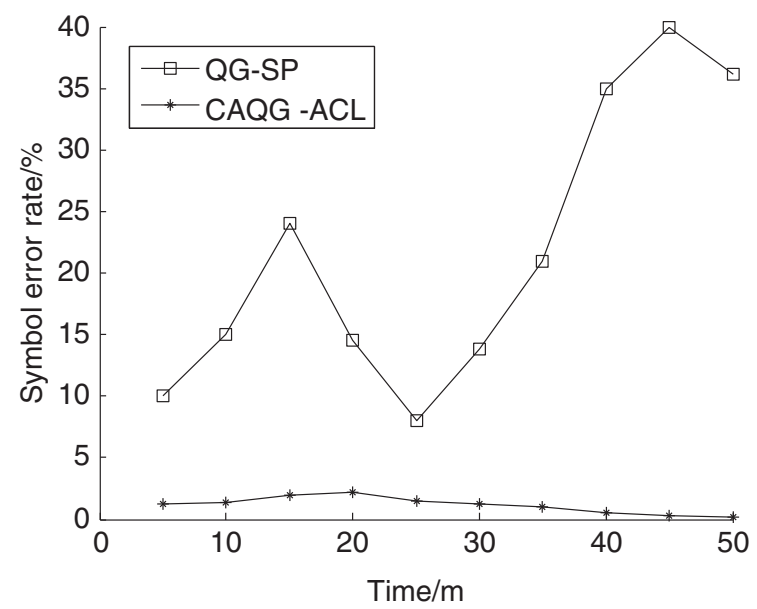

c) Symbol error rate

Fig. 7 Performance analysis of the cloud-assisted QoE guarantee. a Throughput rate. b Execution efficiency. c Symbol error rate 
Simulation results show that the proposed mechanism can not only improve the execution efficiency but also reduce the false symbol rate while maintaining high throughput rate.

\section{Competing interests}

The author declares that he/she has no competing interests.

\section{Acknowledgements}

This work is supported in part by the scientific research project of Changshu Institute of Technology (QZ1403).

Received: 13 November 2015 Accepted: 6 January 2016

Published online: 15 January 2016

\section{References}

1. S Raveau, Z Guo, C Munoz Juan et al., A behavioural comparison of route choice on metro networks: time, transfers, crowding, topology and sociodemographics. Trans. Res Part A-Policy. Practice. 66, 185-195 (2014)

2. Aon M A, Cortassa S. Function of metabolic and organelle networks in crowded and organized media[J]. Frontiers Physiol. 2015;5:523.

3. J Sartuqui, N D' Elia, A Gravina Noel et al., Analyzing the hydrodynamic and crowding evolution of aqueous hydroxyapatite-gelatin networks: digging deeper into bone scaffold design variables. Biopolymers 103(7), 393-405 (2015)

4. E Bilal, T Sakellaropoulos, C Participants et al., A crowd-sourcing approach for the construction of species-specific cell signaling networks. Bioinformatics 31(4), 484-491 (2015)

5. R Agarwal, S Kumar, M Hegde Rajesh, Algorithms for crowd surveillance using passive acoustic sensors over a multimodal sensor network. IEEE. Sensors. J. 15(3), 1920-1930 (2015)

6. H Oh Sang, Classification of imbalanced data using multilayer perceptrons. J. Korea. Contents. Assoc. 9(7), 141-148 (2009)

7. L Castro Cristiano, P Braga Antonio, Novel cost-sensitive approach to improve the multilayer perceptron performance on imbalanced data. IEEE. Trans. Neural. Net. Learn. Syst. 24(6), 888-899 (2013)

8. S Chaudhuri, S Goswami, A Middey, Medium-range forecast of cyclogenesis over North Indian Ocean with multilayer perceptron model using satellite data. Nat. Hazards 70(1), 173-193 (2014)

9. FD Mwale, AJ Adeloye, R Rustum, Application of self-organising maps and multi-layer perceptron-artificial neural networks for streamflow and water level forecasting in data-poor catchments: the case of the Lower Shire floodplain, Malawi. Hydrol. Res. 45(6), 838-854 (2014)

10. C Caylak, I Kaftan, Determination of near-surface structures from multichannel surface wave data using multi-layer perceptron neural network (MLPNN) algorithm. Geophysica 62(6), 1310-1327 (2014)

11. S-A Ouadfeul, V Tourtchine, L Aliouane, Daily geomagnetic field prediction of INTERMAGNET observatories data using the multilayer perceptron neural network. Arabian. J. Geosci. 8(3), 1223-1227 (2015)

12. G Jozanikohan, H Norouzi Gholam, F Sahabi et al., The application of multilayer perceptron neural network in volume of clay estimation: case study of Shurijeh gas reservoir, Northeastern Iran. J. Nat. Gas. Sci. Eng. 22, 119-131 (2015)

13. A Taravat, S Proud, S Peronaci, F Del Frate et al., Multilayer perceptron neural networks model for meteosat second generation SEVIRI daytime cloud masking. Remote. Sens. 7(2), 1529-1539 (2015)

14. H Shafizadeh Moghadam, J Hagenauer, M Farajzadeh et al., Performance analysis of radial basis function networks and multi-layer perceptron networks in modeling urban change: a case study. Int. J. Geographic. Inform. Sci. 29(4), 606-623 (2015)

15. X Fan, S Li, L Tian, Chaotic characteristic identification for carbon price and an multi-layer perceptron network prediction model. Expert. Syst. Appl. 42(8), 3945-3952 (2015)

16. T Patel Krishna, C Stevens Michael, A Meda Shashwath et al., Robust changes in reward circuitry during reward loss in current and former cocaine users during performance of a monetary incentive delay task. Biol. Psychiatry 74(7), 529-537 (2013)

17. L Wei, H Zhu, Z Cao et al., SUCCESS: a secure user-centric and social-aware reputation based incentive scheme for DTNs. AD HOC Sensor. Wire. Net. 19(1-2), 95-118 (2013)
18. T Nadkar, V Thumar, SN Merchant et al., Cognitive relaying with frequency incentive for multiple primary users. Wirel. Pers. Commun. 74(1), 167-187 (2014)

19. G losifidis, L Gao, J Huang et al., Incentive mechanisms for user-provided networks. IEEE Commun. Mag. 52(9), 20-27 (2014)

20. T August, R August, $\mathrm{H}$ Shin et al., Designing user incentives for cybersecurity. Communications ACM 57(11), 43-46 (2014)

\section{Submit your manuscript to a SpringerOpen ${ }^{\mathcal{O}}$ journal and benefit from:}

- Convenient online submission

Rigorous peer review

- Immediate publication on acceptance

- Open access: articles freely available online

- High visibility within the field

- Retaining the copyright to your article

Submit your next manuscript at $\boldsymbol{s p r i n g e r o p e n . c o m ~}$ 